\title{
The combined supplementation of omega-3 fatty acids and probiotics decreased the levels of serum polyamines in experimental colitis
}

\author{
Havvanur YOLDAŞ ÍLKTAÇ ${ }^{1}$, Nihal BÜYÜKUSLU², Cüneyd PARLAYAN
}

Cite this article as:

Yoldaş İlktaç, H., Büyükuslu, N., Parlayan, C. (2021). The combined suplemantation of omega-3 fatty acids and probiotics decreased the levels of serum poyamines in experimental colitis. Food and Health, 7(4), 279-285. https://doi.org/10.3153/FH21029

\footnotetext{
${ }^{1}$ Istanbul Medeniyet University, Faculty

of Health Sciences, Department of

Nutrition and Dietetics, Istanbul,

Turkey

${ }^{2}$ Istanbul Medipol University, Faculty of

Health Sciences, Department of

Nutrition and Dietetics, Istanbul,

Turkey

${ }^{3}$ Bahcesehir University, Faculty of Medicine, Biostatistics and Medical Informatics, Istanbul, Turkey
}

ORCID IDs of the authors:

H.Y.İ. 0000-0002-7433-6370

N.B. 0000-0003-1420-0989

C.P. 0000-0002-6183-9489

Submitted: 21.03 .2021

Revision requested: 02.05 .2021

Last revision received: 03.05 .2021

Accepted: 03.05.2021

Published online: 10.09 .2021

Correspondence:

Havvanur YOLDAŞ İLKTAÇ

E-mail:havvanur.yoldas@medeniyet.edu.tr

\begin{abstract}
Polyamines play an important role in the maintenance of intestinal permeability. Therefore we aimed to determine the effects of probiotics and omega 3 fatty acids on serum polyamine levels in colitis. Fifty BALB/c mice were randomly grouped as normal, colitis with no treatment applied, colitis treated by probiotics (VSL\#3), colitis treated by omega-3, and colitis treated by both probiotics and omega-3. Experimental colitis was induced by injection of $200 \mathrm{mg} / \mathrm{kg}$ 2,4-Dinitrobenzenesulfonic acid (DNBS). The probiotic and the omega-3 fatty acid supplements were applied daily by oral gavage. Serum polyamine levels were measured with high performance liquid chromatography (HPLC). In each group, the levels of serum polyamines are the highest in spermidine and the least in spermine. Bowel inflammation in experimentally induced colitis mice resulted in lower serum polyamine concentrations. In probiotic and omega 3 fatty acid supplemented group significant decreases were observed for spermine and spermidine $(\mathrm{p}<0.001)$, while no significant changes were obtained for putrescine. Combined supplementation of probiotics and omega- 3 fatty acids for 10 days in colitis mice significantly decreased the serum levels of spermine and spermidine.
\end{abstract}

Keywords: Colitis, Inflammatory bowel disease, Omega-3 fatty acids, Probiotics, Polyamines

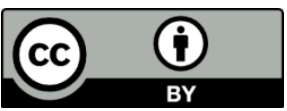

(C) 2021 The Author(s) 


\section{Introduction}

Inflammatory bowel disease (IBD) is a systemic disease associate with an interaction between genetic and environmental factors and intestinal immunologic factors, with no definite etiology. It is mainly classified as ulcerative colitis (UC) and Crohn's disease (CD). Although the etiology of IBD is not fully understood, current studies indicate that the increase of activation in $\mathrm{T}$ cells plays a significant role (Lee et al., 2018). The incidence of UC and CD varies greatly regarding geographical regions and populations and are more common in developed countries. In recent years, the increase in the incidence of the disease in Turkey and other developing countries is noteworthy ( $\mathrm{Ng}$ et al., 2018). The pathogenesis of inflammatory bowel diseases is multifactorial (Lee et al., 2018). There are several theories explaining the possible mechanisms and mediators involved in the progress of the disease. With the impairment of gastrointestinal (GI) mucosal barrier integrity, luminal antigens trigger the mucosal immune system creating tissue damage and thus clinical manifestations of IBD emerge (Ahluwalia et al., 2018). However, it is now widely accepted that IBD is an idiopathic disease caused by a dysregulated immune response to host intestinal microflora (González-Castro et al., 2017). The impaired immune response during infection causes the bacteria to bypass the first line of the immune defence in the host, resulting in increased stimulation of the mucosal immunity system (Shi et al., 2017).

Polyamines; spermidine, spermine, putrescine are aliphatic amines found in both prokaryotic and eukaryotic cells. Polyamines are also produced by bacteria in the intestine. Following the biosynthesis, polyamines are transported into the cell via polyamine carriers. Polyamines have many functions including cell proliferation and immunological response. They also play an important role in the maintenance of intestinal function, the formation of inflammation and the recovery (Hesterberg et al., 2018; Pegg, 2009). The epithelium in the GI system is regulated by passive diffusion by taking the polyamines from extracellular sources or specific polyamine transport proteins (Uemura \& Gerner, 2011). Polyamines have been reported to act as negative immunoregulators on lymphocytes, neutrophil locomotion, natural killer cell activity, and nitrogen oxide production as well as regulatory effects on cell growth and differentiation. It was suggested that the regulatory effect on the inflammatory process arose from the local release of endogenous polyamines after cell formation or injury (Joyce, 2000). Controversial results have been reported in the studies of polyamine metabolism in tissue samples of IBD patients. Moreover, it is demonstrated that deficiency of polyamines are responsible for the impaired intestinal barrier which is an important step in the pathophysiology of IBD. Two factors are responsible for the intestinal barrier disruption. The first, the tight junction increases the basal permeability and causes the barrier defect. Second, epithelial apoptosis or necrosis leads to large epithelial defects leading to local leaks in the intestinal barrier. Bacterial translocation is followed by degradation of the epithelial barrier (Sung, 2015). People with IBD have a deteriorated intestinal bacterial population (Gong et al., 2016). Changes in the bacterial population may cause degradation of polyamine levels. That is why each bacterium can produce polyamines in different amounts. It was shown that the levels of polyamines, especially those with anti-inflammatory properties, are decreased in people with IBD (Weiss et al., 2004). A study on dogs with IBD also revealed a significant decrease in polyamine levels in polyps (Rossi et al., 2018). The elimination of this deficiency is one of the possible mechanisms by which inflammation can be reduced. Polyamines are important for the differentiation and proliferation of the intestinal mucosa that is rapidly renewing itself. Putrescine and spermidine are required for proliferation and spermine is specifically required for differentiation (Gao et al., 2013). Deterioration of polyamine synthesis may cause chronic intestinal inflammation by affecting the epithelial barrier. Currently there is no curative medical tratment for IBD. Alternative treatment methods will be beneficial. Probiotics have a direct effect on the epithelial barrier by increasing mucin, antimicrobial peptides and $\beta$-defensin production in goblet cells, decreasing epithelial permeability to intraluminal pathogens and toxins (Lee et al., 2018). Among the long chain fatty acids, omega3 fatty acids have strong anti-inflammatory properties (Calder, 2012). There are several researches to indicate separately the positive effects of omega- 3 fatty acids and probiotic supplements on IBD treatment (Parker et al., 2018; Scaioli et al., 2017). Our hypothesis is that the combined probiotic and omega-3 fatty acids supplementation affects the levels of serum polyamines in experimentally induced colitis mice. Hence, the detection of the amount of serum polyamines may be useful to monitor the diagnose and treatment of IBD patients. Further studies are needed to examine the association between the levels of serum polyamines and diagnose and progress of IBD. This study was aimed to determine the effects of probiotics and omega 3 fatty acids on serum polyamine levels in colitis.

\section{Materials and Methods}

\section{Study Design}

Fifty BALB/c mice with 20-30 g (6-8 weeks) were used in this study. Animals were obtained from the animal house of 
MEDITAM, Istanbul Medipol University. The animals were housed under controlled conditions. The room temperature was $21 \pm 2^{\circ} \mathrm{C}$ and the air humidity was $50 \pm 5 \%$. The rhythm of light and darkness was established (light phase from 6:00 a.m. to 6:00 p.m.). They had free access to tap water and to standard laboratory chow pellets. Mice were randomly assigned to five groups ( $\mathrm{n}=10$ /group) as healthy control (noncolitis), colitis control, probiotic group (colitis and treated with probiotics), omega-3 (colitis and treated with omega-3), probiotic + omega-3 (colitis and treated with both probiotics and omega-3). All the animals were weighed before colitis induction and before sacrifice.

\section{Induction of Experimental Colitis in Mice}

Before the 2,4-Dinitrobenzenesulfonic acid (DNBS) application mice were fasted for 24 hours and allowed free access to water only. They were intraperitoneally aneshtesized with 80 $\mathrm{mg} / \mathrm{kg}$ body weight of ketamine hydrochloride (Ketalar, Parke Davis ve Eczacibasi, Istanbul) and $10 \mathrm{mg} / \mathrm{kg}$ body weight of xylazine hydrochloride (Rompun, Bayer HealthCare). Colitis was induced by intrarectal injection through this tube of $200 \mathrm{mg} / \mathrm{kg}$ of DNBS solution (Sigma, Aldrich) in $30 \%$ ethanol (EtOH) except normal group as in previous (Martín et al., 2014). Healthy control group received only phosphate-buffered saline (PBS) (Sigma, Aldrich). The same procedure was repeated with the half dose of DNBS $(100 \mathrm{mg} / \mathrm{kg})$ at the 21 th day. The solutions were applied via a $10 \mathrm{~cm}$ of polyurethane cannula placed in $3-4 \mathrm{~cm}$ through rectum. To prevent the reflow the mice were hold upside down for 90 seconds. Afterwith they were kept trandelenburg position untill anesthesia emerged. The protocol of DNBSinduced colitis is detailed in Figure 1 (Martín et al., 2014).

\section{Animal Feed and Nutritional Supports}

Mice were reached the standard chow and water ad libitum whole experimental period. The supplements were given through oral gavage daily between the 14th and 24th days. The equal amount of PBS instead of nutritional supplements were applied to healty and DNBS control groups in the same period. The probiotic support (VSL\#3, Sigma-Tau Pharmaceuticals, Inc.) including Lactobacillus acidophilus, Lb. delbrueckii subsp. bulgaricus, Lb. casei, Lb. plantarum, Bifidobacterium breve, B. longum, B. infantis ve Streptococcus salivarius subsp. thermophilus had $10^{9} \mathrm{CFU} / \mathrm{mL} /$ day [18]. The supplement of omega-3 fatty acid involving $504 \mathrm{mg}$ EPA and $378 \mathrm{mg}$ DHA in a gel capsule (Solgar Inc.) was given $300 \mathrm{mg} / \mathrm{kg} /$ day.

\section{Polyamine Analysis}

At the end of the 24th day, the animals were euthanized using cervical dislocation. Blood specimens were collected through cardiac puncture. For HPLC analysis, blood samples were centrifuged at $15,000 \times g$ at $4{ }^{\circ} \mathrm{C}$ for 10 minutes. After centrifugation, $100 \mu \mathrm{L}$ of the resulting supernatant was removed. A hundred $\mu \mathrm{L}$ of cold $1.5 \mathrm{M} \mathrm{HClO}_{4}$ was added and mixed at medium speed for 30 seconds at $25^{\circ} \mathrm{C}$. Then, $50 \mu \mathrm{L}$ of cold 2 $\mathrm{M} \mathrm{K}_{2} \mathrm{CO}_{3}$ was added, the formed gas was removed. Samples were centrifuged at $15,000 \times g$ for 10 minutes at $4^{\circ} \mathrm{C}$. A hundred $\mu \mathrm{L}$ of supernatant was transferred into a new plastic vial and added $150 \mu \mathrm{L} \mathrm{H}_{2} \mathrm{O}$. Then $700 \mu \mathrm{L}$ of $\mathrm{H}_{2} \mathrm{O}, 50 \mu \mathrm{L}$ of $1.2 \%$ $(\mathrm{w} / \mathrm{v})$ benzoic acid and $50 \mu \mathrm{L}$ of samples were placed in $2 \mathrm{~mL}$ plastic vials for $2 \mu \mathrm{L}$ injection. HPLC analysis was conducted by Waters Alliance e2695 HPLC equipped with Waters Symmetry C18 (75 mm, $3.5 \mu \mathrm{m}, 4.6 \mathrm{~mm})$ analytical column. Waters 2475 FLR detector was used for fluorescent effect of each polyamine derivative with excitation $\lambda$ set at $340 \mathrm{~nm}$ and emission $\lambda$ at $450 \mathrm{~nm}$. As previous study, The Empower 3 software (Waters) was used for HPLC chromatography data analysis (Dai et al., 2014).

\section{Statistical Analysis}

Statistical analysis was performed with SPSS 22.0 package program. All data were tested for normal distribution using the Shapiro-Wilk test and graphical methods. Statistical analyses were performed using ANOVA followed by Hochberg's GT2 or Games-Howell post-hoc test. The results were expressed as mean \pm standard error of the mean (SE) and $p$ values $<0.05$ were considered significant.

\section{Results and Discussion}

The concentrations of natural polyamines, spermine, spermidine and putrescine in all groups are shown in Table 1. The levels of serum polyamines are the highest in spermidine and the least in spermine in both control non-colitis and control colitis groups. However all polyamines decreased in mice with induced colitis in comparison with control non-colitis. In control colitis groups, the only significant differentiation was observed in the level of spermidine $(\mathrm{p}<0.05)$. In comparison with the control colitis mice, the probiotic supplementation decreased the level of spermine and increased the levels of spermidine and putrescine; omega- 3 fatty acids supplementation decreased the levels of spermine and putrescine and increased the level of spermidine; combined probitic and omega-3 fatty acids supplementation decreased the all measured polyamines. Statistical analysis revealed a significant main factor effect of the group supplemented with probiotic and omega- 3 fatty acids (for spermine and spermidine $\mathrm{p}<0.001$; for putrescine $\mathrm{p}<0.005$ ). Combined probiotic and omega-3 fatty acids was administered for 10 days, the spermine values were significantly lower than the values in all other groups $(\mathrm{p}<0.001)$; spermidine values were significantly lower than the values in control non-colitis and omega-3 groups $(p<0.001)$. There were significant differences in serum spermidine values for control colitis, probiotic and the probiotic+omega-3 groups in comparison with control non-colitis group $(\mathrm{p}<0.01)$. The serum putrescine differences were significant 
$(\mathrm{p}<0.01)$ only between the control non-colitis and omega-3 group. We hypothesize that the combined probiotics and omega- 3 fatty acids supplementation in colitis induced mice affects the serum levels of spermine, spemidine and putrescine. Polyamines play key roles in immune response system via T-cell and B-cell development (Hesterberg et al., 2018). The conflicting results from the studies on the relationship between the levels of polyamines and IBD were reported in patients and animals with autoimmune diseases (Park \& Igarashi, 2013; Giacomo Rossi et al., 2015). In isolated colonic epithelial cells from endoscopic biopsies from patients with IBD revealed increased spermidine and $\mathrm{N}^{8}$-acetylspermidine levels reflecting increased uptake and metabolism (Weiss et al., 2004). Nitta et al. (2001) showed that polyamines involved in B cell antigen receptor (BCR) mediated apoptosis, therefore the levels of putrescine, spermidine and spermine were reduced following BCR crosslinking. In a recent review by Hesterberg et al. the role of polyamines in immune cell functions was evaluated (Hesterberg et al., 2018). They reported that BCR-dependent activation induces myeloid cells which regulates $\mathrm{T}$-cell function indicating the role of polyamines autoreactive B- and T-cells in autoimmune disease. Supporting the interaction between polyamine levels and immune system we observed a decrease for polyamines in mice with DNBS induced colitis in comparison with control non-colitis mice. The combined supplement to mice resulted in the minimum spermine, spermidine and putrescine values in comparison with other groups. The dramatic decreases spesifically in the levels of spermine between combined group and others were significant. Studies on humans and animals resulted many beneficial effects of probiotic strains. Linsalata et al. revealed that probiotics reduced the levels of polyamines in normal colonic mucosa of rats (Linsalata et al., 2005). In a similar way, in our study the levels of polyamines in probiotic group were lower than the control non-colitis group. It is known that increasing nutrient uptake contributes to mucosal restoration (Vidal-Lletjós et al., 2017). Daily intake of omega-3 fatty acids are associated with a decrease in inflammation in IBD. A study suggested that any claims of omega-3 fatty acids efficacy in IBD should be regarded with caution (Yakoob \& Abbas, 2016). We observed a significant decrease in the level of putrescine in omega-3 group in comparison with the control non-colitis. However it is yet difficult to conclude that the serum polyamine level is associated with the healthy bowel. Interestingly when probiotic and omega-3 fatty acids were given in combination to mice for 10 days, the levels of polyamines reduced significantly more than the mice which were supplemented with either omega-3 fatty acids or probiotics. In a study, it was shown that the levels of spermine were reduced whereas spermidine was overexpressed in chronic intestinal inflammation (Bailey, 2017). However, Weiss et al. (2004) reported enhanced levels of spermidine and spermine in acute colitis, whereas in chronic inflammation, colonic epithelial cell spermine concentrations were decreased. In our study the levels of spermidine were higher than the levels of spermine and putrescine for each groups, except probiotic+omega-3 and group which the putrescine level was the highest. Moreover, the amounts of serum polyamines decreased in the order of spermidine, putrescine and spermine in each groups except probiotic + omega-3 group.

Table 1. The polyamine concentrations of mice (mean $\pm \mathrm{SE}$ )

\section{Polyamines $(\mathrm{nmol} / \mathrm{mL})$}

Control non-colitis Control colitis Probiotic Omega-3

\section{Probiotic \\ $+$}

Omega-3

\begin{tabular}{ccccccc}
\hline Spermine & $9.3 \pm 0.7$ & $9.2 \pm 0.6$ & $8.8 \pm 0.3$ & $7.8 \pm 0.6$ & $0.3 \pm 0.1^{\mathrm{a}, \mathrm{b}, \mathrm{c}, \mathrm{d}}$ & 0.000 \\
Spermidine & $20.0 \pm 1.7$ & $11.6 \pm 0.9^{\mathrm{a}}$ & $11.8 \pm 1.5^{\mathrm{a}}$ & $14.1 \pm 1.4$ & $6.8 \pm 0.8^{\mathrm{a}, \mathrm{d}}$ & 0.000 \\
Putrescine & $10.3 \pm 0.2$ & $9.3 \pm 0.2$ & $10.2 \pm 1.3$ & $8.1 \pm 0.2^{\mathrm{a}}$ & $7.7 \pm 0.6$ & 0.005 \\
\hline
\end{tabular}

Values expressed as mean \pm SE; Except in control group, all other mice were induced with DNBS for colitis.

${ }^{a} \mathrm{p}<0.01$ vs control non-colitis; ${ }^{b} \mathrm{p}<0.01$ vs control colitis; ${ }^{\mathrm{c}} \mathrm{p}<0.01$ vs probiotic; ${ }^{\mathrm{d}} \mathrm{p}<0.05$ vs omega-3 group. 


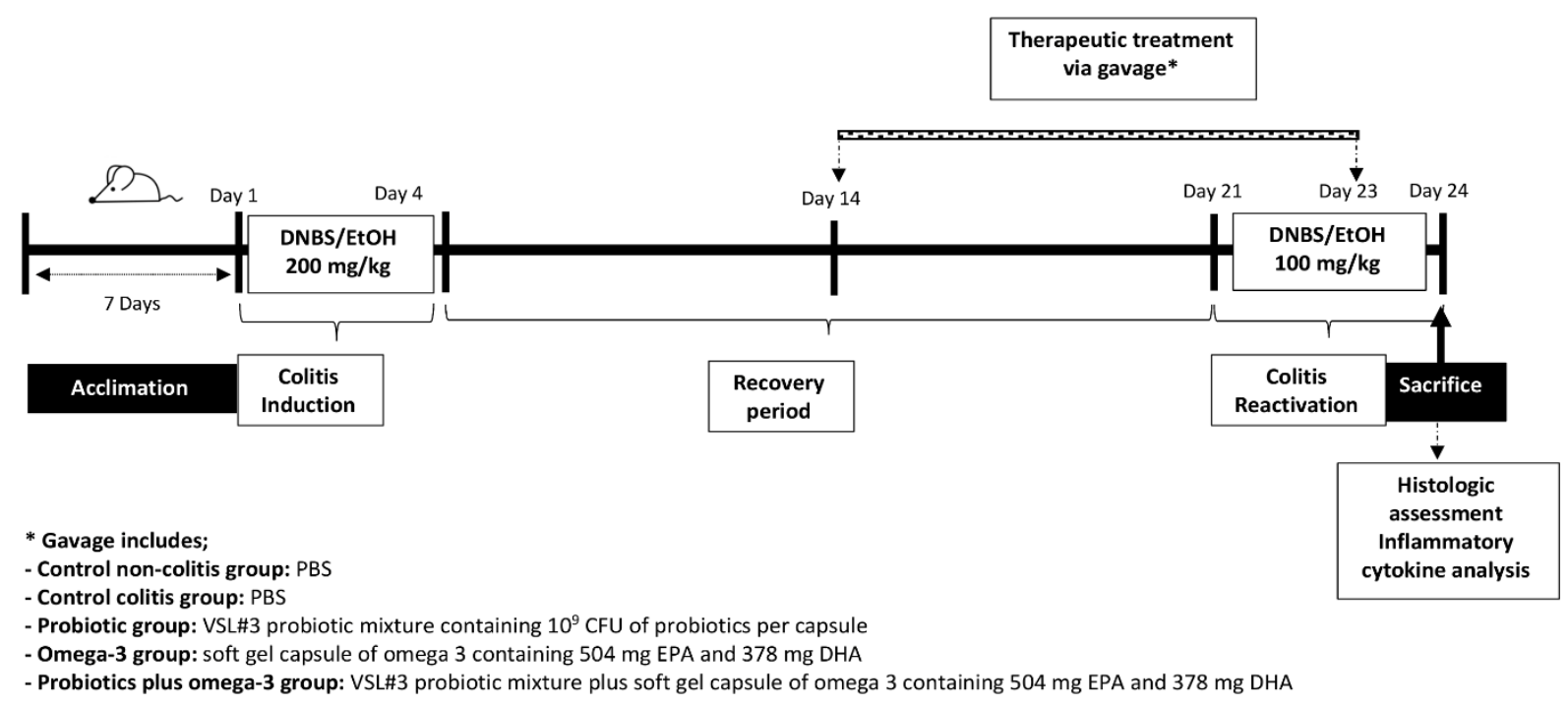

Figure 1. Experimental protocol.

Figure 1. Experimental protocol

\section{Conclusion}

This study revealed that the combined probiotics and omega3 fatty acids supplementation in colitis induced mice decreased the serum levels of spermine, spemidine and putrescine more than in the mice fed with either one of these supplements. However the levels of polyamines in colitis mice groups did not reached the levels as in the healthy mice. It seems that the inflammation of bowel decreases polyamine levels irreversibly. Further research is needed to evaluate the interactions of probiotic microorganisms combined with omega-3 fatty acids in terms of understanding of metabolic pathways.

\section{Compliance with Ethical Standard}

Conflict of interests: The authors declare that there is no conflict of interests.

Ethics committee approval: The study received ethical approval from the Experimental Animal Research Committee of Istanbul Medipol University (12/2014, no. 38328770/83).

Funding disclosure: -

Acknowledgments: -

Disclosure: - 


\section{References}

Ahluwalia, B., Moraes, L., Magnusson, M. K., Öhman, L. (2018). Immunopathogenesis of inflammatory bowel disease and mechanisms of biological therapies. Scandinavian Journal of Gastroenterology, 53(4), 379-389.

https://doi.org/10.1080/00365521.2018.1447597

Bailey, J. (2017). PTH-082 Dysregulated polyamines in the inflammatory bowel disease gut-a novel therapeutic target. Gut, 66(2), A247.

Calder, P.C. (2012). Long-chain fatty acids and inflammation. Proceedings of the Nutrition Society, 71(2), 284-289.

https://doi.org/10.1017/S0029665112000067

Dai, Z., Wu, Z., Wang, J., Wang, X., Jia, S., Bazer, F. W., Wu, G. (2014). Analysis of polyamines in biological samples by HPLC involving pre-column derivatization with ophthalaldehyde and N-acetyl-L-cysteine. Amino acids, 46(6), 1557-1564.

https://doi.org/10.1007/s00726-014-1717-z

Gao, J.-H., Guo, L.-J., Huang, Z.-Y., Rao, J., Tang, C.W. (2013). Roles of cellular polyamines in mucosal healing in the gastrointestinal tract. Journal of Physiology and Pharmacology: An Official Journal of the Polish Physiological Society, 64, 681-693.

Gong, D., Gong, X., Wang, L., Yu, X., Dong, Q. (2016). Involvement of Reduced Microbial Diversity in Inflammatory Bowel Disease. Gastroenterology Research and Practice, 2016, 6951091.

https://doi.org/10.1155/2016/6951091

González-Castro, A. M., Martínez, C., Salvo-Romero, E., Fortea, M., Pardo-Camacho, C., Pérez-Berezo, T., Alonso-Cotoner, C., Santos, J., \& Vicario, M. (2017). Mucosal pathobiology and molecular signature of epithelial barrier dysfunction in the small intestine in irritable bowel syndrome. Journal of Gastroenterology and Hepatology, 32(1), 53-63.

https://doi.org/10.1111/jgh.13417

Hesterberg, R.S., Cleveland, J.L., Epling-Burnette, P.K. (2018). Role of Polyamines in Immune Cell Functions. Medical Sciences (Basel, Switzerland), 6(1), 22.

https://doi.org/10.3390/medsci6010022
Joyce, S. (2000). Natural T cells: Cranking up the immune system by prompt cytokine secretion. Proceedings of the National Academy of Sciences, 97(13), 6933-6935.

https://doi.org/10.1073/pnas.97.13.6933

Lee, S.H., Kwon, J.E., Cho, M.-L. (2018). Immunological pathogenesis of inflammatory bowel disease. Intestinal Research, 16(1), 26-42.

https://doi.org/10.5217/ir.2018.16.1.26

Lee, S.H. (2015). Intestinal permeability regulation by tight junction: implication on inflammatory bowel diseases. Intestinal Research, 13(1), 11-18.

https://doi.org/10.5217/ir.2015.13.1.11

Linsalata, M., Russo, F., Berloco, P., Valentini, A., Caruso, M., Simone, C., Barone, M., Polimeno, L., Di Leo, A. (2005). Effects of Probiotic Bacteria (VSL\#3) on the Polyamine Biosynthesis and Cell Proliferation of Normal Colonic Mucosa of Rats. In Vivo (Athens, Greece), 19, 989995.

Martín, R., Chain, F., Miquel, S., Lu, J., Gratadoux, J.-J., Sokol, H., Verdu, E. F., Bercik, P., Bermúdez-Humarán, L. G., Langella, P. (2014). The commensal bacterium Faecalibacterium prausnitzii is protective in DNBS-induced chronic moderate and severe colitis models. Inflammatory Bowel Diseases, 20(3), 417-430.

https://doi.org/10.1097/01.MIB.0000440815.76627.64

Ng, S.C., Shi, H.Y., Hamidi, N., Underwood, F.E., Tang, W., Benchimol, E.I., Panaccione, R., Ghosh, S., Wu, J.C.Y., Chan, F.K.L., Sung, J.J.Y., Kaplan, G.G. (2018). Worldwide incidence and prevalence of inflammatory bowel disease in the 21st century: a systematic review of population-based studies. Lancet (London, England), 390(10114), 2769-2778.

https://doi.org/10.1016/S0140-6736(17)32448-0

Nitta, T., Igarashi, K., Yamashita, A., Yamamoto, M., Yamamoto, N. (2001). Involvement of polyamines in B cell receptor-mediated apoptosis: spermine functions as a negative modulator. Experimental Cell Research, 265(1), 174-183.

https://doi.org/10.1006/excr.2001.5177 
Park, M.H., Igarashi, K. (2013). Polyamines and their metabolites as diagnostic markers of human diseases. Biomolecules \& Therapeutics, 21(1), 1-9.

https://doi.org/10.4062/biomolther.2012.097

Parker, E.A., Roy, T., D'Adamo, C.R., Wieland, L.S. (2018). Probiotics and gastrointestinal conditions: An overview of evidence from the Cochrane Collaboration. Nutrition, 45, 125-134.e11.

https://doi.org/https://doi.org/10.1016/j.nut.2017.06.024

Pegg, A.E. (2009). Mammalian polyamine metabolism and function. IUBMB Life, 61(9), 880-894.

https://doi.org/10.1002/iub.230

Rossi, G., Cerquetella, M., Scarpona, S., Pengo, G., Fettucciari, K., Bassotti, G., Jergens, A. E., \& Suchodolski, J. S. (2018). Effects of probiotic bacteria on mucosal polyamines levels in dogs with IBD and colonic polyps: a preliminary study. Beneficial Microbes, 9(2), 247255.

https://doi.org/10.3920/BM2017.0024

Rossi, G., Cerquetella, M., Pengo, G., Mari, S., Balint, E., Bassotti, G., Manolescu, N. (2015). Immunohistochemical expression of ornithine decarboxylase, diamine oxidase, putrescine, and spermine in normal canine enterocolic mucosa, in chronic colitis, and in colorectal cancer. BioMed Research International, 2015, 1-8.

https://doi.org/10.1155/2015/172756

Scaioli, E., Liverani, E., Belluzzi, A. (2017). The imbalance between n-6/n-3 polyunsaturated fatty acids and inflammatory bowel disease: A comprehensive review and future therapeutic perspectives. International Journal of Molecular Sciences, 18(12).

https://oi.org/10.3390/ijms18122619

Shi, N., Li, N., Duan, X., Niu, H. (2017). Interaction between the gut microbiome and mucosal immune system. Military Medical Research, 4, 14.

https://doi.org/10.1186/s40779-017-0122-9

Uemura, T., Gerner, E. W. (2011). Polyamine transport systems in mammalian cells and tissues. Methods in Molecular Biology (Clifton, N.J.), 720, 339-348.

https://doi.org/10.1007/978-1-61779-034-8_21

Vidal-Lletjós, S., Beaumont, M., Tomé, D., Benamouzig, R., Blachier, F., Lan, A. (2017). Dietary protein and amino acid supplementation in inflammatory bowel disease course: What impact on the colonic mucosa? Nutrients, 9(3).

https://doi.org/10.3390/nu9030310

Weiss, T. S., Herfarth, H., Obermeier, F., Ouart, J., Vogl, D., Schölmerich, J., Jauch, K.-W., Rogler, G. (2004). Intracellular polyamine levels of intestinal epithelial cells in inflammatory bowel disease. Inflammatory Bowel Diseases, 10(5), 529-535.

https://doi.org/10.1097/00054725-200409000-00006

Yakoob, J., Abbas, Z. (2016). Role of omega-3 fatty acids in irritable bowel Synd rome (IBS). international Journal of Pharmacological Research, 6, 271-277. 